\title{
Smartphones or laptops in the collaborative classroom? A study of video-based learning in higher education
}

\author{
This paper explores how the use of smartphones vs. laptops influences students' \\ engagement, behaviour and experience watching academic videos in a \\ collaborative classroom. Experiments were run in authentic teaching sessions \\ with a total of 483 first-year higher education students. The methodology applied \\ is a quasi-experimental design with post-test-only, being the independent \\ variable, the device used to visualize the academic videos. Results indicate that \\ the use of laptops have provided better results in terms of student's engagement \\ with the videos, their collaborative behaviour and satisfaction with the device. \\ Hence, the findings of this research suggest that the type of mobile device used in \\ activities that consider the use of videos in a collaborative class need to be \\ carefully chosen to maximize the student's comfortability - and in consequence, \\ their engagement with the video-based learning activity and their positive \\ behaviour and experience within the collaborative context.
}

Keywords: video-based learning; computer-supported collaborative learning; higher education; human-computer interaction; smartphones; laptops

\section{Introduction}

Despite the use of videos has a long history in education, the popularity of Video-Based Learning (VBL) has increased as a result of new forms of online education, most noticeably in the case of Massive Open Online Courses (Giannakos 2013; Yousef, Chatti and Schroeder 2014). VBL has unique features that make it an effective Technology-Enhanced Learning approach which can improve learning outcomes as well as learner satisfaction given its flexibility and motivational potential (Yousef, Chatti and Schroeder 2014). Yet, the mere use of videos in class is not an improvement (neither a pedagogy) by itself. Videos are resources that should be considered as supporting material in the application of active teaching and learning methodologies (Blomberg et al. 2014) that are aligned with the requirements of a specific learning situation, 
considering desired learning goals and the target learner group (Blomberg et al. 2014;

Seidel, Blomberg and Renkl 2013).

In this line, Masats and Dooly (2011) provide a series of coherently integrated video activities to guide future teachers in the design of VBL scenarios. According to the way in which videos are used, authors describe four categories in which VBL experiences can be grouped: video-viewing, video-modelling, video-coaching and video-making. As these experiences (especially those including video-viewing) do not necessarily need to happen in the presence of the educator, their application has led to blended learning designs where the visualization of videos is proposed to be done outside the classroom and active face-to-face (f2f) activities are suggested for the classroom. This approach has been recently termed as the 'Flipped Classrooms' (FC) or inverted classrooms-, as an instance of the VBL approach that enables saving inclassroom time devoted to the explanation of concepts (Tucker 2012). In the FC model, learners watch video lectures as homework. The class is then turned into an active learning session where the teacher proposes the use of case studies, labs, games, simulations, or experiments to discuss the concepts presented in the video lecture (Herreid and Schiller 2013). In many cases, it is showed that the result of introducing videos in a learning design eventually converges in a FC model. But other researchers have noticed that other models are possible. For example, it is also possible to use videos in a hands-on class as a support tool that encourages a more autonomous, flexible and significant learning during the realisation of practical activities (Albó et al. 2016). In addition, with the advances in the technology, higher education is experiencing a change in how VBL resources are delivered (Maniar et al. 2008). Students can visualize the videos with their computers at class or at home but also have the opportunity to do so with their mobile devices: tablets, smartphones, laptops etc. at 
any place and time. So, with the use of mobile devices, potential learning occurs regardless of location: beyond the space of a brick and mortar course or even beyond the space inside an online course management system (Gikas and Grant 2013).

Yet, on the captious side, research shows that the use of mobile devices in the classroom can be an element of distraction for learning, can reduce quality of $f 2 f$ interactions and hands-on skills, or create dependency (Anshari et al. 2017). For instance, the use of smartphones in class facilitate multitasking (Grinols and Rajesh 2014), what some studies associate with a decrease of the students' academic performance (Jacobsen and Forste 2011; Lepp, Barkley and Karpinski 2015). Moreover, some scholars state that mobile phone screens are too small to provide a comfortable learning environment (Wang and Shen 2012). In a study investigating whether the screen size constrains video-based mobile learning, Maniar et al. (2008) reported that physical screen size of a mobile device does influence the learning experience. For example, learners' satisfaction regarding their learning experience differed based on the screen size: in the large and medium screen conditions, students' responses tended to be positive whereas the students' responses in the small screen condition were not entirely positive. Their findings were in agreement with other researchers who suggested that screen size is critical to the success of effective learning (Papanikolaou and Movromoustakos 2006).

More recent research problematizes the situation (questioning the current validity of previous research), claiming that we, humans, are getting more used to the use of small screens to support daily-life activities and that smartphones are gaining space in the educational ecosystem as educators and students are showing more and more positive attitudes towards them (Al-Emran, Elsherif, and Shaalan, 2016; BrizPonce et al. 2017; Byrne-Davis et al. 2015; Cheon et al. 2012; Chung, Chen and Kuo 
2015; Abachi and Muhammad 2014). There is evidence that the use of this technology in class can provide benefits affecting potentially effective learning, such students' motivation, enjoyment, flexibility, consistency, engagement, convenience (Conradie, Lombard and Moller 2013; Papanikolaou and Movromoustakos 2006; So 2016). While the use of small screens is still recognized to be challenging in the delivery of entire courses, researchers reckon that they are a good option to offer short learning activities (e.g., those based on the visualization of videos) if content is adapted to these devices (Alamri et al. 2014).

Therefore, there is a need for further research investigating how the use of different types of devices can impact learning scenarios that employ videos (Conradie, Lombard and Moller 2013; Lepp, Barkley and Karpinski 2015) using different pedagogies and learning designs (Conradie, Lombard and Moller 2013; Gedik et al. 2012; Wang and Shen 2012). This paper is focused on this research line, in particular it investigates the impact of using smartphones in comparison to the use of laptops to support VBL activities in collaborative classroom scenarios within the engineering teaching context. Whereas there are some studies in the Computer-Supported Collaborative Learning literature that explore the relevance of the group size in a groupbased mobile learning (Melero, Hernández-Leo and Manatunga 2015) and whether interactive groupware interfaces can support small group work in classrooms (Clayphan et al. 2016), no previous study has investigate how the use of different mobile devices influences the students' engagement, behaviour and experience watching academic videos in a collaborative classroom. These types of scenarios have been also underexplored in the VBL literature. Yet, they are especially challenging and interesting, given the collaborative nature of the task and the potential for using one or several devices within a group, and the research questions they trigger around the 
effects that these types of the devices (smartphones vs. laptops) can cause in student behaviour in this context (Kukulska-Hulme et al. 2009).

The structure of the paper is as follows. Section 2 details the hypothesis of the study and section 3 describes the methodology. Results are presented in section 4, followed by a discussion in section 5 . Section 6 closes the paper with the main conclusions of the research.

\section{Purpose of current study}

The purpose of this study is to investigate how the use of different mobile devices influences students' engagement, behaviour and experience watching academic videos. Specifically, it aims to compare the use of smartphones versus the use of laptops in the context of the visualization of videos to support hands-on in-class activities. In order to address this objective, six hypotheses have been stated based on the previous research mentioned above.

Regarding the engagement of students for visualizing the videos:

- H1.1. Students using laptops (given their bigger screen sizes) will visualize more videos than those using smartphones.

- H1.2. The number of video visualizations of the students using smartphones will increase through the three years. As students are becoming more used to using mobile devices and the performance of this type of device improves over time.

Regarding the behaviour of the students' visualizing the videos:

- H2.1. Students using laptops will tend to visualize the videos completely (full duration) whereas students using smartphones will tend to stop watching videos before they finish. 
- H2.2. Students using laptops will tend to visualize the videos in group whereas students using smartphones will tend to visualize the videos individually.

Regarding the students' experience watching the videos:

- H3.1. Students using smartphones would prefer using another device for watching videos more than the students using laptops.

- H3.2. Students using laptops will find videos more useful than students using smartphones.

\section{Methodology}

This research was conducted using a quasi-experimental design with post-test-only (Creswell 2002). This methodology was considered appropriate because the objective of this investigation was to study a cause-and-effect relationship: whether the use of different mobile devices influences the student's engagement, behaviour and experience watching academic videos. We were able to manipulate the independent variable, the device allowed for visualizing the academic videos, which had two categories: smartphone and laptop, to analyse the different outcomes or dependent variables: the effects predicted in the six hypotheses described above in the cause-and-effect equations.

\subsection{Participants, sample and treatment conditions}

Participants were the students of the course 'Introduction to ICT' of the academic years 2014-2015, 2015-2016 and 2016-2017 (Moreno and Hernández-Leo 2014). This course is a mandatory subject for first-year students of the bachelor's degrees in Computer Engineering, Telecommunications Network Engineering and Audiovisual Systems Engineering at Engineering School of a Spanish university. The course is quarterly and 
offers a global overview of the university, its resources, the chosen degrees, an introduction to transversal skills and the professional field of ICT engineering. Introduction to ICT has 6 ECTS (European Credit Transfer and Accumulation System) credits corresponding to 150 hours of student work, of which 50 are in class sessions. These 50 hours are divided into lectures ( 28 hours), medium-group sessions ( 8 hours) and seminar sessions with a small group of students (14 hours).

Specifically, the setting for this research was a 2-hour f2f seminar session, of the mentioned course, focused on explicitly addressing the transversal skills of teamwork and oral communication. This seminar implies an individual reading of an article related to problems that typically appear in teamwork; a critical reflection in teams about several questions proposed by the teacher; and finally, an oral presentation in which each team must share with the other classmates the key take-away points of the session. Each seminar session had an average 14 students and participants worked in teams of 35 people.

In the first edition, which corresponds to the academic year 2014-2015, 147 students participated in the experiment. They were divided in 12 seminars resulting in 35 working teams. In the second edition (2015-2016), we had 167 students divided as well as in 12 seminars resulting in 43 teams. Finally, in the third edition (2016-2017), 169 students participated in the study within 11 seminars and 50 teams. This study considers the aggregated data of the three editions therefore the final sample is 483 students - 35 seminars and 128 teams (see Total column in Table 1). The allocation of students to seminar sessions is randomly done by the university secretariat. Team formation within each seminar was freely done by the students. Thus, a quasiexperimental design was considered. 
Table 1. Participants' sample of the experiment.

\begin{tabular}{|c|c|c|c|c|c|c|c|c|c|c|}
\hline Ed. & $\begin{array}{c}\text { Academic } \\
\text { year }\end{array}$ & \multicolumn{3}{|c|}{ Laptops group (Freq.) } & \multicolumn{2}{c|}{ Smartphones group (Freq.) } & \multicolumn{3}{c|}{ Total (Freq.) } \\
\cline { 3 - 12 } & Seminars & Teams & Students & Seminars & Teams & Students & Seminars & Teams & Students \\
\hline 1 & $2014-15$ & 6 & 16 & 65 & 6 & 19 & 82 & 12 & 35 & 147 \\
\hline 2 & $2015-16$ & 6 & 23 & 95 & 6 & 20 & 72 & 12 & 43 & 167 \\
\hline 3 & $2016-17$ & 5 & 21 & 73 & 6 & 29 & 96 & 11 & 50 & 169 \\
\hline
\end{tabular}

In order to manipulate the treatment conditions, the seminars were divided in two groups randomly: laptops group (during the seminar students were allowed to access the videos only using laptops) and smartphones group (during the seminar students were allowed to access the videos only using smartphones). Table 1 shows the frequencies of participants in each experimental group by academic year. In total, of the 483 students, 233 were in the laptops group condition whereas 250 were in the smartphones group.

\subsection{Procedure}

Each seminar consisted of a two hours activity about how to improve team work and oral communications skills. This was divided in three main students' tasks (see Fig.1):

(1) Reading (individual - $10 \mathrm{~min}$ ): each student had to read a short article about the main challenges of teamwork. The teacher delivered copies of the article to the students on paper together with the description of the seminar activity.

(2) Collaborative discussion and presentation preparation (in teams $-40 \mathrm{~min}$ ): the first 20 minutes students had to work in teams and discuss possible answers to five questions regarding the article - which were posted by the teacher in the seminar description document. During the last 20 minutes of this task, each team had to summarize the key points of the discussion preparing a four-seven 
minutes presentation. To support the discussion and prepare the presentation, the teacher recommended watching four academic videos: two of them were related to how to support teamwork (useful to find answers to the proposed questions related to the article read); whereas the other two were related to the oral communication skills (with hints about how to prepare a good oral presentation). Watching the recommended videos was optional: students could decide by themselves whether watching the videos (and which ones and when) or not. The videos lasted between 1.5 and 4.5 minutes. Watching the whole four videos would take about 12 minutes (out of 40 minutes which would require the activity - the $30 \%$ of the total time allocated for doing the activity).

(3) Presentation (in teams $-60 \mathrm{~min}$ ): each team had to do the final presentation (4 min) - in which the participation of all the team members was mandatory - in front of all the other students of the seminar. Finally, teacher gave feedback to each team after their presentations.

At the beginning of the session, before starting the three activities described above, the teacher showed in a classroom projection where they could find the videos. Moreover, she wrote on the board the name of the recommended videos for the session. She also highlighted that all the videos had subtitles and transcriptions in the three official languages of the university (Catalan, Spanish and English). Finally, she asked students to form the teams (between three and five people). The videos used in the experiment were recorded and edited by a team of technicians from the university together with the academic team of the subject. The translations of the videos were done by a professional translator and reviewed by two experts in the content ensuring that the quality of the videos was the same regardless of the language. 
Some days before the seminars, the teacher sent an email to the students asking them to bring their own devices to that particular seminar session. Moreover, the teacher always had three auxiliary laptops in case there were teams without their own devices. At the beginning of the session, after introducing the videos, teacher asked participants to only use one device to visualize them (laptop or smartphone, depending on the condition associated to the particular seminar). Students did not know what the activity would be until they got to class to ensure that no participant was more prepared than other. In addition, the two groups received the same introduction at the beginning of the activity. Finally, all teams from all seminars and experiment groups (laptops and smartphones) had as minimum one device available for participating on the activity. Table 2 shows the number of available devices per teams depending on the device used and experiment edition (data obtained by the observations in class, described in the next section of this article).

Table 2. Number of available devices per teams depending on the experimental group.

\begin{tabular}{|l|c|c|c|c|c|c|c|c|c|}
\hline Ed. & $\begin{array}{c}\text { Academic } \\
\text { year }\end{array}$ & \multicolumn{3}{|c|}{ Laptops group } & \multicolumn{3}{c|}{ Smartphones group } \\
\cline { 3 - 10 } & $\begin{array}{c}\text { Teams } \\
\text { (Freq.) }\end{array}$ & $\begin{array}{c}\text { Students } \\
\text { per Team } \\
\text { (in average) }\end{array}$ & $\begin{array}{c}\text { Available } \\
\text { devices } \\
\text { (Freq.) }\end{array}$ & $\begin{array}{c}\text { Available } \\
\text { devices per } \\
\text { Team } \\
\text { (in average) }\end{array}$ & $\begin{array}{c}\text { Teams } \\
\text { (Freq.) }\end{array}$ & $\begin{array}{c}\text { Students } \\
\text { per Team } \\
\text { (in average) }\end{array}$ & $\begin{array}{c}\text { Available } \\
\text { devices } \\
\text { (Freq.) }\end{array}$ & $\begin{array}{c}\text { Available } \\
\text { devices per } \\
\text { Team } \\
\text { (in average) }\end{array}$ \\
\hline 1 & $2014-15$ & 16 & 4,0 & 18 & 1,2 & 19 & 4,3 & 51 & 2,7 \\
\hline 2 & $2015-16$ & 23 & 4,1 & 41 & 1,8 & 20 & 3,6 & 46 & 2,3 \\
\hline 3 & $2016-17$ & 21 & 3,5 & 36 & 1,7 & 29 & 3,3 & 63 & 2,2 \\
\hline
\end{tabular}




\subsection{Instrumentation, data collection, analysis and threads to validity}

This study used three instruments to gather the data from the field: an observation protocol, automatic registers from the videos in YouTube and a questionnaire (see Figure 1).

Task 1. Reading (10min, individual)
Task 2. Discussion and presentation preparation (40min, in teams)

\section{- Observation}

-Youtube analytics
Task 3.

Presentation

(60min, in teams)

-Questionnaire

Figure 1. Main tasks of the two hours seminar and their associated research instruments.

During each seminar, at least one researcher was observing the participants

whereas they were doing the second task, with the main objective of observing how

students were interacting with the videos through the devices (laptops or smartphones).

The observers participating in this study were trained by the main researchers using an

observation protocol. This served as a guide during the observations since it divided the occurrences to observe depending on its priority: high, medium or low (see Table 3).

Table 3. List of occurrences to observe depending on its priority.

\begin{tabular}{|c|c|}
\hline $\begin{array}{l}\text { Level of } \\
\text { priority }\end{array}$ & Occurrence to observe \\
\hline \multirow[t]{2}{*}{ High } & $\begin{array}{l}\text { How students were watching the videos: } \\
\text { - } \quad \text { Individually } \\
\text { - } \quad \text { With one or more members of the working team } \\
\text { - } \quad \text { With pairs } \\
\text { - } \\
\text { - } \\
\text { - }\end{array}$ \\
\hline & $\begin{array}{l}\text { Level of concentration during the visualization: } \\
\text { - } \quad \text { They watch the entire video in silence } \\
\text { - } \quad \text { They discuss the video whereas they are watching it } \\
\text { - } \quad \text { Others }\end{array}$ \\
\hline Medium & $\begin{array}{l}\text { The interaction between the different working teams: } \\
\text { - They discuss or share information about the videos with the members of } \\
\text { other working teams }\end{array}$ \\
\hline
\end{tabular}




\begin{tabular}{|l|c|}
\hline \multirow{2}{*}{} & $-\quad$ They share doubts with the members of other teams \\
& $-\quad$ Others \\
\hline Low & $\begin{array}{r}\text { Interaction between the students and the teacher: } \\
-\quad \text { What type of questions they ask to the teacher } \\
\end{array}$ \\
& $-\quad$ Related with the content \\
& $-\quad$ Related with doubts about the activity's performance \\
\hline
\end{tabular}

All the visualizations were registered by the video hosting service (YouTube) including the date, time, location and the device used to visualize the videos. Furthermore, at the end of the activity, teacher delivered a questionnaire to gather data from the participants regarding their use of the videos during the activity. In particular, the most relevant questions were the following:

(1) How many videos did you visualize?

(2) Have you watched all the videos completely? If you have not watched or watched only some of the videos, specify the reason.

(3) Would you like to watch the videos in another device? (Yes. Which? / No / I don't know)

(4) How did you organize yourselves within the team to watch the videos? (Using a single device to watch the videos all together / Using more than one device equally distributed / Each team member watched the videos on their own)

(5) Indicate your level of agreement with the following statement (1 being lowest and 4 being highest): I found the videos useful to develop the session's task.

Participants were assured that data collection, storage, and reporting would guarantee confidentiality and anonymity; they gave their informed consent for participation. The analysis of the data was done by using mixed methods design with convergent parallel design (Creswell 2002). The statistical test chosen was a group comparison statistic. Specifically, the Mann-Whitney U test was used to examine differences in engagement, behaviour and experience between students using laptops and those using smartphones 
(as the sample was not normally distributed). Moreover, an ANOVA test was performed to examine the differences between the three editions of the experiment regarding the number of visualizations in the case of the smartphones group. All the statistics were implemented using SPSS software for Windows (IBM SPSS Statistics, version 23).

Triangulation was used to compensate the threats that the quasi-experimental approach introduces to internal validity (Creswell 2002). Furthermore, since observers may become more experienced during time, it was used a strong observation protocol and training. However, despite the external validity is major in quasi-experiment research than in true experiments (as it occurs in real contexts rather than artificial approaches) (Creswell 2002) and the sample used was high (483 students), it has to be taken into account that this study has been implemented within a specific context that add limitations to the generalization of the results: participants were engineering students of a first year graduate program in a public Spanish university.

At the same time, this sample's homogeneity allowed a better control for characteristics of participants that might influence the relationship between the independent and dependent variables minimizing the external factors in the experiment. Other extraneous variables identified (as can be the time of the seminar's sessions and the type of classrooms used) were affecting equally to whole sample. All the seminars were happening in random time slots during the morning (between the 8:30am and the $2: 30 \mathrm{pm})$ as well as both experimental groups had the seminars randomly in three different type of classrooms: with fix tables and chairs, with mobile tables and chairs and with mobile chair-desks. Finally, the teacher in charge was the same person for all seminars during all the three editions. She participated as an additional researcher in the experiment and helped in monitoring the process closely so that the threats to internal validity were minimized. 


\section{Results}

The results obtained in this study are analysed considering three different perspectives:

a) students' engagement watching the videos (i.e. number the videos watched per student/working team depending on the device used); b) their behaviour watching the videos (i.e. duration of the visualizations or working teams' organization); and their experience's valuation (i.e. satisfaction with the device used or the perception of the videos' usefulness).

\subsection{Students' engagement watching the videos}

An indicator of students' engagement with the use of videos is the number of videos they (decided to and) actually watched during the activity. Figure 2 shows the results from the questionnaires regarding the number of videos watched by the students depending on the device used. Results indicate that students using laptops ( $\mathrm{n}=233$, mean $=2.24, \mathrm{sd}=1.623)$ watched more videos than students using smartphones $(\mathrm{n}=250$, mean $=1.39, \mathrm{sd}=1.493$ ) - students using laptops watched 2.24 videos (in average) whereas students using smartphones watched 1.39 videos (the significance of this result was checked using the Mann-Whitney U test with a resulting $\mathrm{p}<0.05$, Cohen's $\mathrm{d}=0.54$ ). The major differences can be seen at the extremes of the graph where $44.0 \%$ of the students using smartphones did not watch any video whereas in the case of the laptops group it is only $25.3 \%$. However, $36.9 \%$ of the students using laptops watched the four recommended videos while in the case of the smartphones group it was only the $16.0 \%$. 


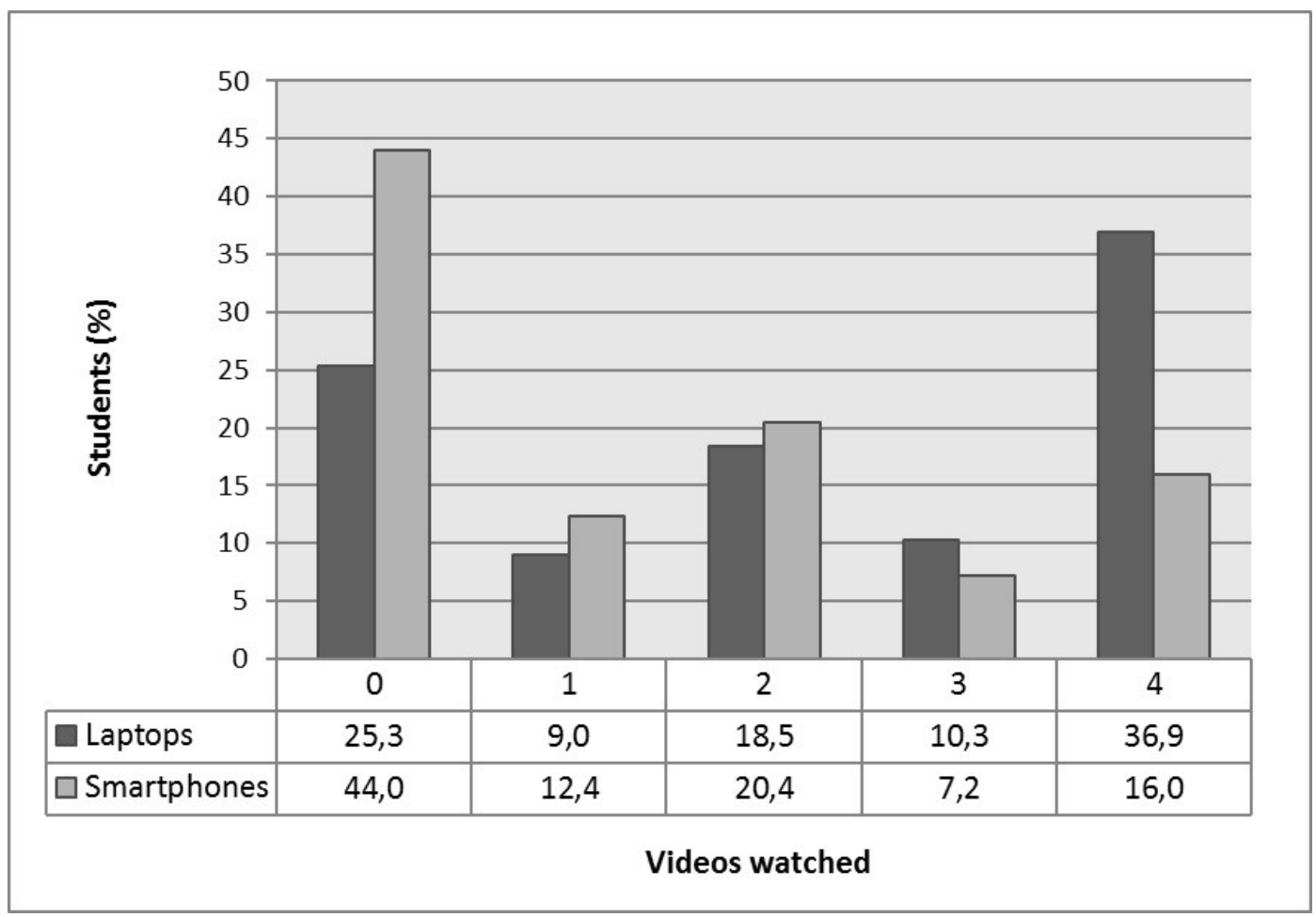

Figure 2. Number of videos watched by the students depending on the device used.

Results from the questionnaire: Laptops, $\mathrm{n}=233$, mean $=2.24, \mathrm{sd}=1.623$ - Smartphones, $\mathrm{n}=250$, mean=1.39, $\mathrm{sd}=1.493$ (Mann-Whitney $\mathrm{U}, \mathrm{p}<0.05$, Cohen's $\mathrm{d}=0.54$ ).

The above finding is supported by the results obtained from the observations in class. In Figure 3 it can be seen the number of videos watched by the teams depending on the device used. Similar than in the previous results, teams using laptops were observed watching more videos than those using smartphones (means of 1.70 and 1.09 respectively, being a significant result, checked using the Mann-Whitney U test with a resulting $\mathrm{p}<0.05$, Cohen's $\mathrm{d}=0.48$ ). According to these direct observations by researchers, $45.6 \%$ of the teams that were using smartphones did not watch any video whereas this was only observed in $25.9 \%$ of the teams using laptops. However, $33.3 \%$ of the teams using laptops watched more than two videos in contrast to the $13.3 \%$ observed in the case of teams using smartphones. 


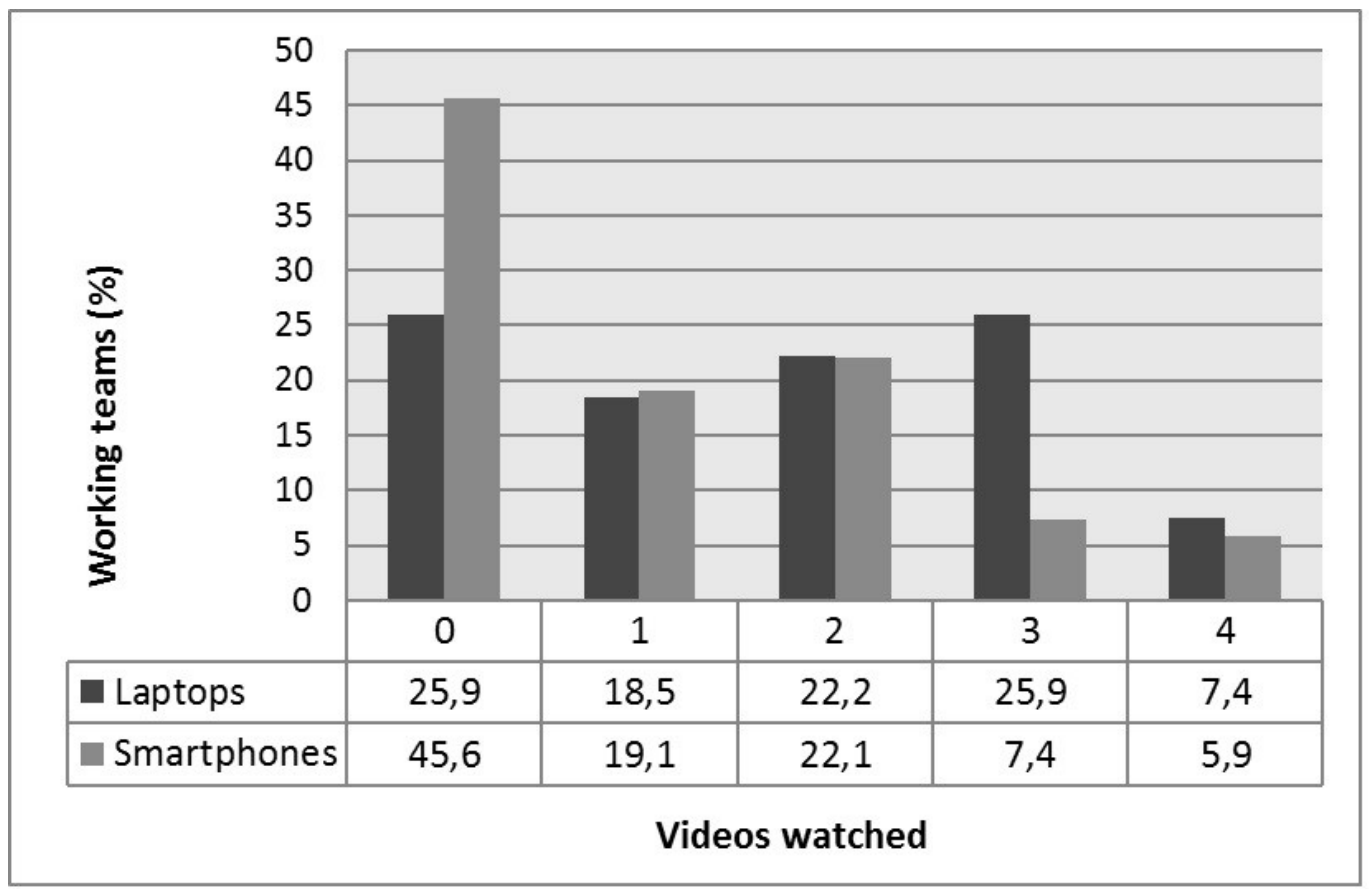

Figure 3. Number of videos watched from the working teams depending on the device used. Results from the observations: Laptops, $\mathrm{n}=54$, mean=1.70, $\mathrm{sd}=1.312-$ Smartphones, $\mathrm{n}=68$, mean=1.09, $\mathrm{sd}=1.231$ (Mann-Whitney $\mathrm{U}, \mathrm{p}<0.05$, Cohen's $\mathrm{d}=0.48)$.

Data obtained from YouTube analytics was not considered for comparing the number of videos watched depending on the device used with the above results from the other sources (observations and questionnaire' responses) because it was not possible to distinguish if visualizations were individual or in teams. Despite this limitation, if we look at the number of total views (referring to all videos) during the laptops' sessions, there were 190 views; whereas during the smartphones' sessions there were 140 views. Moreover, if we look at the visualization time, the overall minutes students watched in total was 384 minutes during the laptops' seminars whereas in the smartphones group the overall minutes watched from the all videos were 296 minutes. 
When participants were asked in the questionnaire about the reasons why they did not watch the videos, most of the participants who did not watch any video stated that it was because they were prioritizing preparing the task itself (an oral presentation) instead of watching the videos $(80 \%$ in the case of laptops group and $53 \%$ in the smartphones group). The second reason expressed in both groups was that they did not find necessary the visualization of the videos to perform the task $(10 \%$ in the case of laptops group and $15 \%$ in the smartphones group). Of those using smartphones, the third reason expressed $(13 \%)$ was related to having technical problems for watching the videos (especially with the Internet connection). Moreover, $9 \%$ of them stated that they forgot watching the videos because they were focused on doing the activity. Other reasons that were given in the case of the smartphones group include: they did not find the device comfortable enough to watch the videos, they found the subtitles were too small to read (even more when they were sharing the device), they did not have headphones and that the screen was too small.

Within the smartphones group, it is interesting to visualize the number of videos watched in average depending on the academic year (Figure 4) in order to check whether this number has been increased as the hypothesis H1.2 suggested. Despite results show a trend of increasing numbers of videos watched per student through the years (sample sizes of 82, 72 and 96; means of 1.24, 1.32 and 1.56; standard deviation of $1.384,1.471$ and 1.595$)$, the result is not statistically significant (checked using an ANOVA test with a resulting $\left.\mathrm{p}>0.05, \mathrm{\eta}^{2}=0.009\right)$. This result was supported by the data obtained from the observations in class and the YouTube analytics. 


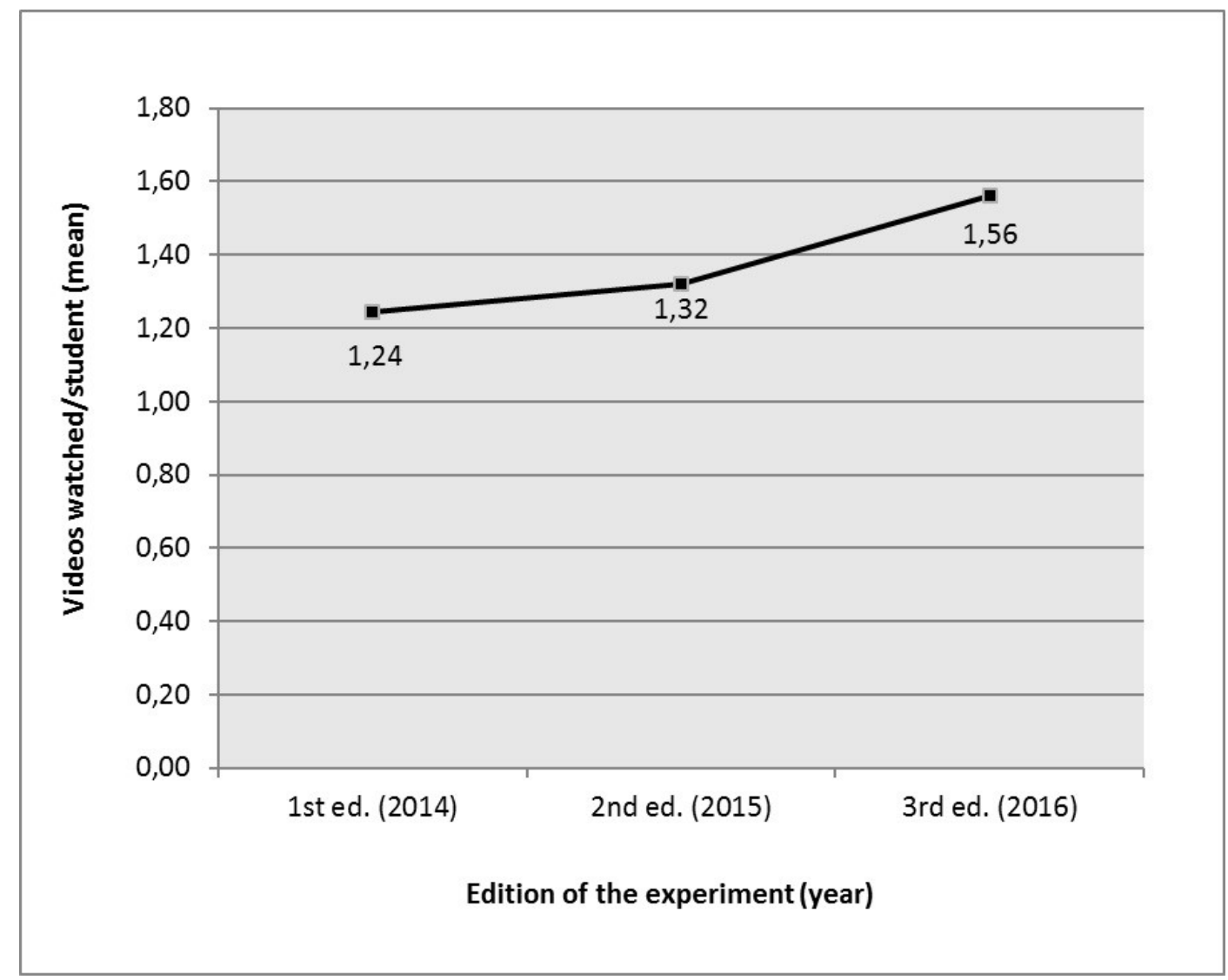

Figure 4. Mean of number of videos watched per student within the smartphones group depending on the experiment's edition (academic year). Results from the questionnaire: $\mathrm{n} 1=82, \mathrm{n} 2=72, \mathrm{n} 3=96\left(\right.$ ANOVA $\left.\mathrm{p}>0.05, \mathrm{n}^{2}=0.009\right)$.

\subsection{Students' behaviour watching the videos}

The questionnaire included two questions regarding student's behaviour watching the videos. The first one was about the completeness of the visualizations, whether they had watched the videos from the beginning to the end or just part of the videos. In particular, the question was 'Have you watched all the videos completely?' and the possible answers to choose were 'No', 'Only some of them' and 'Yes, all of them'. Results are presented in Figure 5 and the difference between the means for the two experimental groups was statistically significant (tested using Mann-Whitney U test, $\mathrm{p}<0.05$, Cohen's $d=0.44)$ : students using laptops $(\mathrm{n}=172$, mean $=2.56, \mathrm{sd}=0.623$ ) 
visualized more videos in their whole duration than those using smartphones $(n=138$, mean $=2.28, \mathrm{sd}=0.637)$.

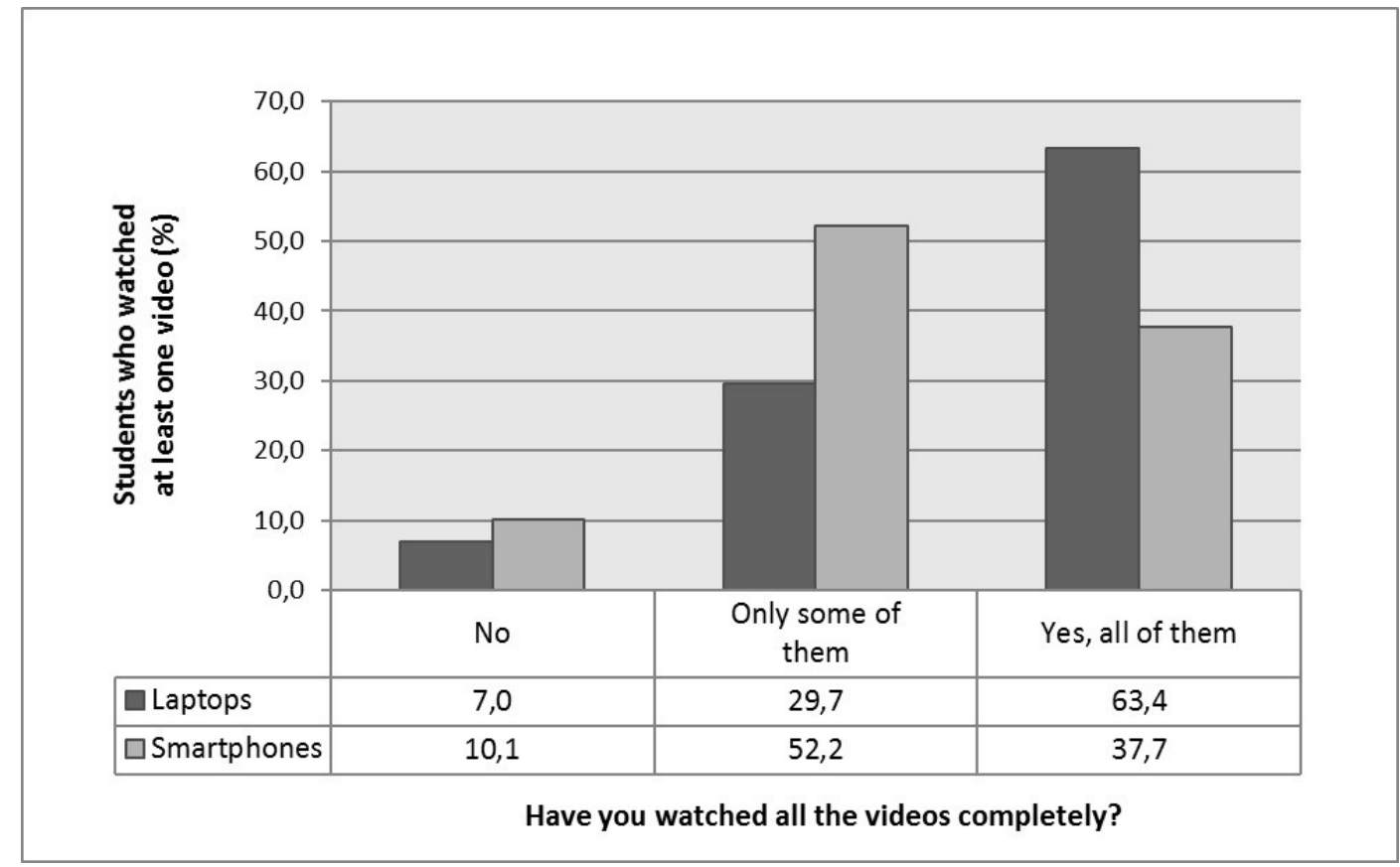

Figure 5. Frequency of videos watched completely depending on the device used.

Results from the questionnaire: Laptops, $\mathrm{n}=172$, mean $=2.56, \mathrm{sd}=0.623$ - Smartphones, $\mathrm{n}=138$, mean $=2.28, \mathrm{sd}=0.637$ (Mann-Whitney $\mathrm{U}, \mathrm{p}<0.05$, Cohen's $\mathrm{d}=0.44$ ).

Most of the students using laptops (63.4\%) stated that they watched all the videos completely while most of the students in the smartphones group (52.2\%) only watched completely some of them. In any case, somehow surprisingly, there was an important percentage of students using smartphones (37.7\%) that watched all the videos completely.

The second question related to the organization of the teams when it came to visualize the videos. Results indicate that there is a significant difference between the laptops and the smartphones groups (checked with the Mann-Whitney $U$ test, $p<0.05$, Cohen's $\mathrm{d}=0.34$ ). The first tend to watch the videos in teams (sharing the device/s) 
whereas in the second this happens in much less frequently (see Figure 6). The different answers were coded in order to compare the means of the two groups as follows: one device per working team was coded with a 1; more than one device per working team with a 2; and one device per each member of the team with a 3 . The resulting means were 1.29 in the laptops group $(\mathrm{sd}=0.537)$ and 1.51 in the smartphones group $(\mathrm{sd}=0.744)$. Despite the difference, a somehow surprising percentage of students using smartphones (64.3\%) shared one device between 3-5 people.

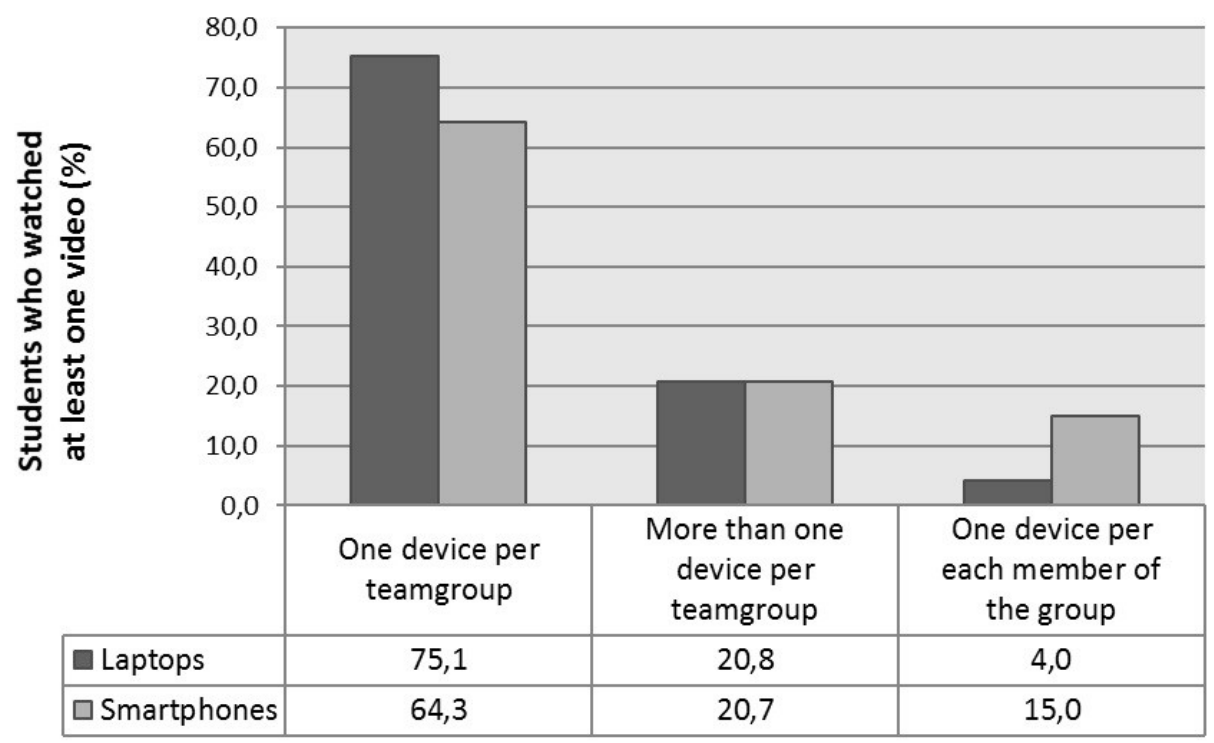

Teamgroup organization for watching the videos

Figure 6. Working teams' organization for watching the videos depending on the device used. Results from the questionnaire: Laptops, $\mathrm{n}=173$, mean $=1.29, \mathrm{sd}=0.537-$ Smartphones, $\mathrm{n}=140$, mean=1.51, $\mathrm{sd}=0.744$ (Mann-Whitney $\mathrm{U}, \mathrm{p}<0.05$, Cohen's $\mathrm{d}=0.34)$.

Above results were supported by the results obtained from classroom observations, being the differences between the experimental groups statistically 
significant in both cases as well (the completeness of the visualizations and the teams' organization for watching the videos).

\subsection{Students' experience watching the videos}

In order to evaluate the students' experience of using videos during the activity, they had the opportunity to express whether they would prefer using another device than the laptop or the smartphone (depending on the group) in the questionnaire. Moreover, they could indicate whether they found the videos useful to develop the second task.

Regarding the preference of using another device, results show a significant difference between the two groups (see Figure 7). More than half of the students that used laptops (55.5\%) stated that they would not have preferred using another device for watching the videos whereas $23.1 \%$ of them stated that they would. The numbers are reversed for the case of students that used smartphones: $26.6 \%$ of them indicated they liked the device used to watch the videos and would not have preferred using another device, but $49.6 \%$ expressed their preference of using another device. The answers to the question 'Would you like to watch the videos in another device?' were coded as follows: 'No' with a 1, 'I do not know it' with a 2 and 'Yes' with a 3. Statistically, there was a significant difference between the two group (mean=1.68, $\mathrm{sd}=0.828$ in laptops group; and mean $=2.23, \mathrm{sd}=0.845$ in the smartphones one) tested with the MannWhitney U test ( $<<0.05$, Cohen's d=0.66). 


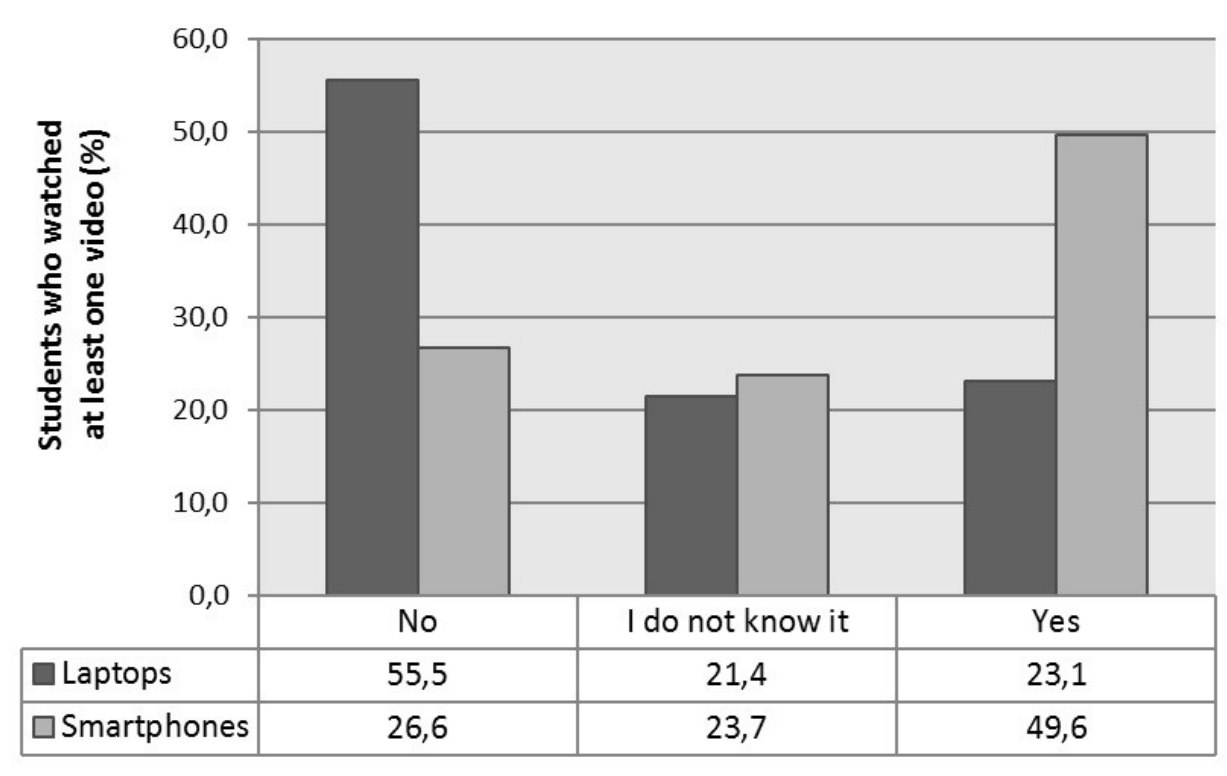

Would you like to watch the videos in another device?

Figure 7. Preference for watching the videos with another device depending on the device used. Results from the questionnaire: Laptops, $n=173$, mean $=1.68, \mathrm{sd}=0.828-$ Smartphones, $\mathrm{n}=139$, mean=2.23, $\mathrm{sd}=0.845$ (Mann-Whitney $\mathrm{U}, \mathrm{p}<0.05$, Cohen's $\mathrm{d}=0.66)$.

Of those $23.1 \%$ that used laptops and would have preferred watching the videos in another device: $74 \%$ would have preferred watching the video with the class projector, $22 \%$ with a smartphone and $4 \%$ with a tablet. Of those $49.6 \%$ that used smartphones and would have preferred watching the videos in another device: $44 \%$ would have preferred watching the video with a laptop/pc, $39 \%$ with a class projector and $17 \%$ with a tablet.

When participants were asked about their level of agreement with the statement 'I found the videos useful to develop the session's task', results were more positive in the case of the laptops group (see Figure 8). Half of the participants (51\%) that used laptops chose the highest level (4) and 40\% chose the third level (3). Regarding the results of the smartphones group, only $35 \%$ chose the maximum level (4) of agreement 
while $43 \%$ chose the third one (3). Moreover, $22 \%$ of the students using smartphones expressed low levels of agreement ( 1 or 2$)$ while in the case of those that used laptops this only happened for the $9 \%$ of the students.

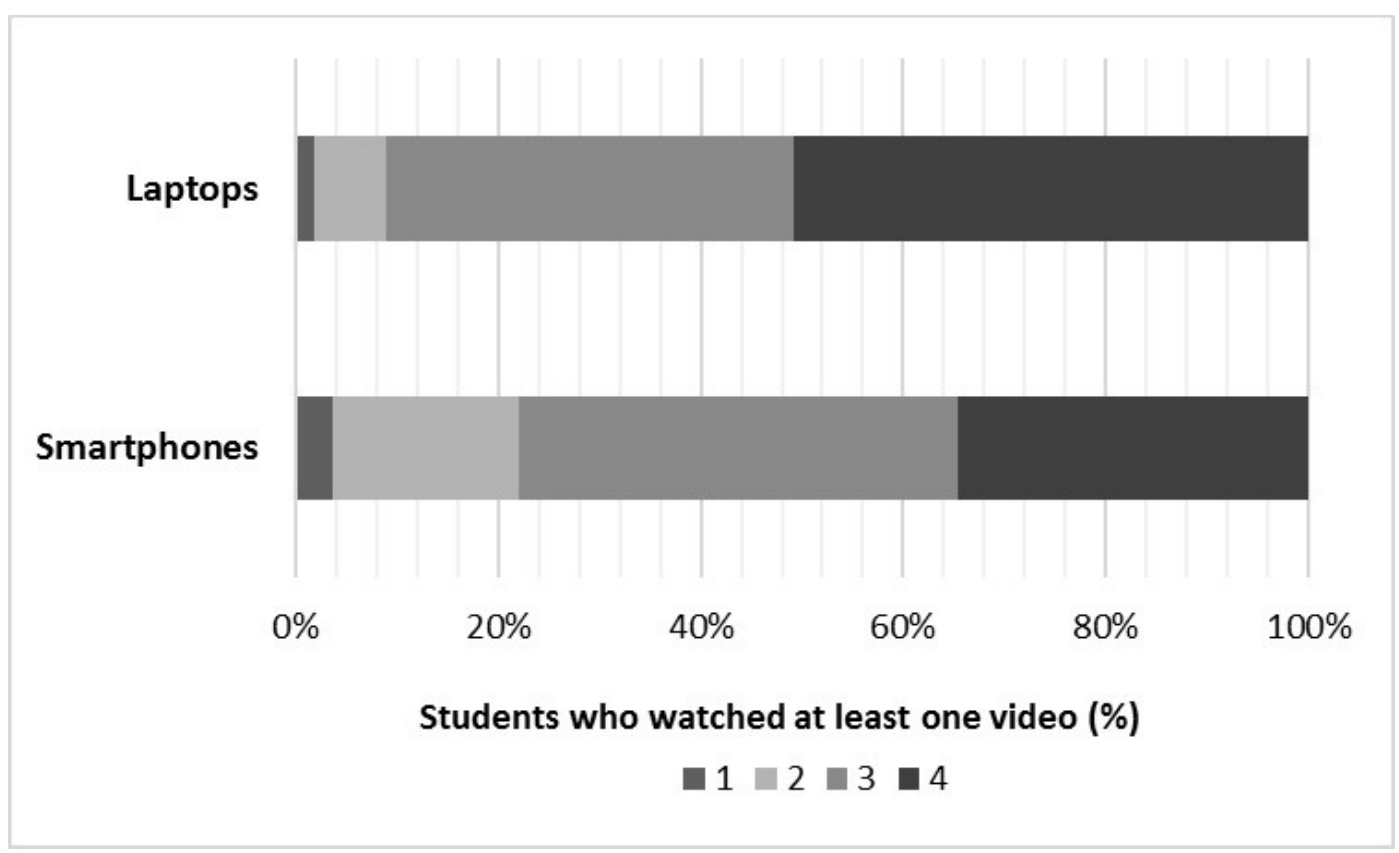

Figure 8. Level of agreement with the statement 'I found the videos useful to develop the session's task' ( 1 being the lowest and 4 being the highest). Results from the questionnaire: Laptops, $\mathrm{n}=169$ - Smartphones, $\mathrm{n}=136$.

Despite these significant differences, results indicate a general satisfaction regarding the utility of videos to develop the session's task.

\section{Discussion}

Results reported above support the assumption that the type of mobile device used for watching the videos (laptops or smartphones) affects participants' engagement, behaviour and overall collaborative VBL classroom experience. It was hypothesized that participants using laptops would be more engaged with the videos than those using smartphones. This hypothesis (H1.1) is confirmed, as the number of visualizations of 
the students that used laptops was significantly higher than those that used smartphones. Comments from participants suggest that having a bigger screen than a smartphone (i.e. laptops' screens), offers more comfort for watching audio-visual content, especially in collaborative learning contexts. Despite this result, it is important to note that $25 \%$ of the students in the laptops group decided not to watch videos, mainly due to time constraints. Hence, results suggest that the activities that involve the use of videos must be carefully designed in order to provide sufficient time flexibility for the students to take advantage of this type of resources. These results are in line with Masats and Dooly (2011, 1159), who stated that 'the way in which the video use is embedded within other tasks is relevant to the eventual teaching and learning'.

The study shows a trend of increasing numbers of visualizations in smartphones (H1.2) along the three academic years in which this experimentation was conducted. However, the difference between the numbers of videos' visualizations along the experiment editions (academic years) is not statistically significant. Further work extending the experimentation along additional academic years would help to better understand whether the smartphones comfortability (in terms of screen size and network connections) will increase in the next years - affecting more significantly students' engagement. Since other researchers pointed out, the screen size can be a problem due to the human visual perception limits, as the attention can be affected depending on the small detail it can be seen (Chen et al. 2003).

Results confirm hypotheses H2.1 and H2.2. Data shows that the type of mobile device used for watching the videos affects students' behaviour during the visualizations. Students that watched the videos using laptops tended to visualize them more completely and in teams than those that used smartphones. A possible explanation for this may be (again) the comfortability of the mobile device, especially related with 
the size of their screen (usually, being the laptops' screens bigger than the smartphones' screens). However, the percentages of students in the smartphones' groups that watched all the videos completely (37.7\%) and were sharing their devices $(64.3 \%$, one device per team; $20.7 \%$, more than one device per team) were somehow surprisingly high. Although it may seem difficult to share a smartphone between 4 or 5 people, students found strategies to do it - for instance, physically supporting the smartphone on top of a case (or any other object) and raising the video's volume (or activating the subtitles). Another common case observed was the smartphone shared by a couple of students that also were sharing headphones (a headset cable for each). In fact, the video's audio was a challenge for both experimental groups. While listening videos with a high volume allowed sharing devices, it was at the same time an inconvenient for the other teams in the class (as the different teams' audios could interfere between them making it difficult content understanding for each team). The solutions that students found were (mainly) two: the use of headphones, which it was restricting the sharing of the device to only two students; or the use of subtitles, which in the case of laptops is easier as their screen was bigger. Therefore, as it was suggested by Wang and Shen $(2012,571)$, 'multimedia mobile content should be carefully chosen to cover all the possible circumstances of the learner'. Nevertheless, results point out that (for now) laptops are a better option than smartphones in case of collaborative activities implying visualization of videos.

Finally, results also confirm hypotheses H3.1 and H3.2. With respect to hypothesis H3.1, it was found that students with smartphones would prefer using another device to watch the videos (more than those using laptops) - especially laptops or projectors. The type of device used also affected students' perception of videos' usability. Students that felt more comfortable with their device (laptops group) found the videos more useful for developing the proposed task (H3.2). Therefore, it can be 
stated that the type of the mobile device used during the collaborative activity actually influenced the students' learning experience. Results are aligned with Maniar et al. (2008) who state that firstly, physical screen size of a mobile device does influence learning. And secondly, and even more notable, that the perception of usability can have a direct influence on the acceptance of mobile learning.

\section{Conclusions}

The aim of the present research was to investigate how the use of different mobile devices (specifically, laptops versus smartphones) influences students' engagement, behaviour and experience when watching academic videos to support collaborative learning activities in the classroom. The study has shown that the use of laptops provides more positive results (than when using smartphones) in terms of students' engagement with the videos, collaborative behaviours and the overall Video-Based Learning experience. The findings suggest that the type of mobile device used in collaborative learning activities that consider the use of videos need to be carefully chosen in order to maximize students' and groups' comfortability. However, the results also show positive trends along the academic years regarding the potential of smartphones to reach important levels of engagement and user satisfaction. Future research should continue monitoring this evolution as well as consider similar experimentation in the frame of other contexts, pedagogies and learning designs that apply VBL.

\section{Acknowledgements}

This research was partly funded by the Spanish Ministry of Science (TIN2014-53199-C3-3-R and TIN2017-85179-C3-3-R), the Maria de Maeztu Units of Excellence Programme (MDM2015-0502), RecerCaixa (CoT project) and the UPF PlaQUID2012. Authors want to thank all the participants, collaborators and researchers who participated in the experiment. 


\section{References}

Abachi, H. R, and G. Muhammad. 2014. "The impact of m-learning technology on students and educators." Computers in Human Behavior 30: 491-496. doi:10.1016/j.chb.2013.06.018

Al-Emran, M., H. M. Elsherif, and K. Shaalan. 2016. "Investigating attitudes towards the use of mobile learning in higher education." Computers in Human Behavior 56: 93-102. doi:10.1016/j.chb.2015.11.033.

Alamri, A., G. Muhammad, A. A. Al Elaiwi, K. N. Al-Mutib, and M. S. Hossain. 2014. "Media content adaptation framework for technology enhanced mobile elearning." Journal of Universal Computer Science 20 (15): 2016-2023.

Albó, L., D. Hernández-Leo, L. Barceló, and L. Sanabria. 2016. "Video-based Learning in Higher Education: The Flipped or the Hands-On Classroom?" Special Issue of the European Journal of Open, Distance and E-Learning (Best of EDEN 2015) $50-61$.

Anshari, M., M. N. Almunawar, M. Shahrill, D. K. Wicaksono, and M. Huda. 2017. "Smartphones Usage in the Classrooms: Learning Aid or Interference?" Education and Information Technologies 22 (6): 3063-3079. doi:10.1007/s10639-017-9572-7.

Blomberg, G., M. G. Sherin, A. Renk1, I. Glogger, and T. Seidel. 2014. "Understanding video as a tool for teacher education: Investigating instructional strategies to promote reflection." Instructional Science 42 (3): 443-463. doi:10.1007/s11251013-9281-6.

Briz-Ponce, L., A. Pereira, L. Carvalho, J. A. Juanes-Méndez, and F. J. García-Peñalvo. 2017. "Learning with mobile technologies - Students' behavior." Computers in Human Behavior 72: 612-620. doi:10.1016/j.chb.2016.05.027.

Byrne-Davis, L., H. Dexter, J. Hart, T. Cappelli, G. Byrne, I. Sampson, and J. Mooney. 2015. "Just-in-time research: A call to arms for research into mobile technologies in higher education." Research in Learning Technology 23. doi:10.3402/rlt.v23.25653.

Chen, L., X. Xie, X. Fan, W. Y. Ma, H. J. Zhang, and H. Q. Zhou. 2003. "A Visual Attention Model for Adapting Images on Small Displays." Multimedia Systems 9 (4): 353-64. doi:10.1007/s00530-003-0105-4. 
Cheon, J., S. Lee, S. M. Crooks, and J. Song. 2012. “An investigation of mobile learning readiness in higher education based on the theory of planned behavior." Computers \& Education 59: 1054-1064. doi:10.1016/j.compedu.2012.04.015.

Chung, H. H., S. C. Chen, and M. H. Kuo. 2015. “A study of EFL college students' acceptance of mobile learning." Procedia - Social and Behavioral Sciences 176: 333-339. doi:10.1016/j.sbspro.2015.01.479.

Clayphan, A., R. Martinez-Maldonado, M. Tomitsch, S. Atkinson, and J. Kay. 2016. "An in-the-wild study of learning to brainstorm: comparing cards, tabletops and wall displays in the classroom." Interacting with Computers 28 (6): 788-810. doi:10.1093/iwc/iww001.

Conradie, P., A. Lombard, and M. Moller. 2013. 'Learners' Perceptions of Mobile Devices for Learning in Higher Education - Towards a Mobile Learning Pedagogical Framework." World Academy of Science, Engineering and Technology, International Science Index 73, International Journal of Social, Behavioral, Educational, Economic, Business and Industrial Engineering 73 (1): 120-125. http://scholar.waset.org/1307-6892/5288

Creswell, J. W. 2002. Educational research: Planning, conducting, and evaluating quantitative and qualitative research. Upper Saddle River, New Jersey: Pearson/Merrill Prentice Hall.

Gedik, N., A. Hanci-Karademirci, E. Kursun, and K. Cagiltay. 2012. "Key instructional design issues in a cellular phone-based mobile learning project." Computers and Education 58 (4): 1149-1159. doi:10.1016/j.compedu.2011.12.002.

Giannakos, M. N. 2013. "Exploring the video-based learning research: A review of the literature.” British Journal of Educational Technology 44 (6): 191-195. doi:10.1111/bjet.12070.

Gikas, J., and M. M. Grant. 2013. "Mobile computing devices in higher education: Student perspectives on learning with cellphones, smartphones \& social media.” The Internet and Higher Education 19: 18-26. doi:10.1016/j.iheduc.2013.06.002.

Grinols, A. B., and R. Rajesh. 2014. "Multitasking with Smartphones in the College Classroom.” Business Communication Quarterly 77 (1): 89-95. doi:10.1177/2329490613515300. 
Herreid, C. F., and N. A. Schiller. 2013. "Case Studies and the Flipped Classroom.” Journal of College Science Teaching 42 (5): 62-66. http://www.jstor.org/stable/43631584

Jacobsen, W. C., and R. Forste. 2011. "The wired generation: academic and social outcomes of electronic media use among university students." Cyberpsychology, Behavior and Social Networking 14 (5): 275-280. doi:10.1089/cyber.2010.0135.

Kukulska-Hulme, A., M. Sharples, M. Milrad, I. Arnedillo-Sánchez, and G. Vavoula. 2009. "Innovation in Mobile Learning: A European Perspective.” International Journal of Mobile and Blended Learning 1 (1): 13-35. doi:10.4018/jmbl.2009010102.

Lepp, A., J. E. Barkley, and A. C. Karpinski. 2015. "The Relationship Between Cell Phone Use and Academic Performance in a Sample of U.S. College Students." SAGE Open 5 (1): 1-9. doi:10.1177/2158244015573169.

Maniar, N., E. Bennett, S. Hand, and G. Allan. 2008. "The effect of mobile phone screen size on video based learning." Journal of Software 3 (4): 51-61. doi:10.4304/jsw.3.4.51-61.

Masats, D., and M. Dooly. 2011. "Rethinking the use of video in teacher education: A holistic approach." Teaching and Teacher Education 27 (7): 1151-1162. doi:10.1016/j.tate.2011.04.004.

Melero, J., D. Hernández-Leo, and K. Manatunga. 2015. “Group-based mobile learning: Do group size and sharing mobile devices matter?" Computers in Human Behavior 44: 377-385. doi:10.1016/j.chb.2014.11.078.

Moreno, V., and D. Hernández-Leo. 2014. "Using academic videos: how do the students perceive them? The experience on 'Introduction to the ICTs."' In VIII CIDUI. Flexible training models: a response to the current needs. Tarragona, Spain.

Papanikolaou, K., and Movromoustakos, S. 2006. "Critical Success Factors for the Development of Mobile Learning Applications." In Proceedings of the IASTED International Conference on Internet, Multimedia Systems and Applications, as part of the $24^{\text {th }}$ IASTED International Multi-Conference on Applied Informatics, edited by A. Boucouvalas, 19-24. Innsbruck, Austria: IASTED/ACTA Press.

Seidel, T., G. Blomberg, and A. Renkl. 2013. "Instructional strategies for using video in teacher education." Teaching and Teacher Education 34: 56-65. doi:10.1016/j.tate.2013.03.004. 
So, S. 2016. "Mobile instant messaging support for teaching and learning in higher education." The Internet and Higher Education 31: 32-42. doi:10.1016/j.iheduc.2016.06.001.

Tucker, B. 2012. “The flipped classroom.” Education Next, 12: 82-83.

Wang, M., and R. Shen. 2012. "Message design for mobile learning: Learning theories, human cognition and design principles." British Journal of Educational Technology 43 (4): 561-575. doi:10.1111/j.1467-8535.2011.01214.x.

Yousef, A. M. F., M. A. Chatti, and U. Schroeder. 2014. "Video-Based Learning: A Critical Analysis of The Research Published in 2003-2013 and Future Visions." In eLmL 2014, The Sixth International Conference on Mobile, Hybrid, and Online Learning, 112-119. Barcelona, Spain: IARIA XPS Press. 